\title{
Fibroepithelial Breast Tumors in a Teenager with Beckwith-Wiedemann Syndrome: A Case Report and Review of Literature
}

\author{
(1) Ayşenur Oktay ${ }^{1}$, (D) Habib Ahmad Esmat ${ }^{2}$, (1) Özge Aslan ${ }^{1}$ \\ ${ }^{1}$ Department of Radiology, Ege University, İzmir, Turkey \\ ${ }^{2}$ Department of Radiology, Kabul University of Medical Sciences, Kabul, Afghanistan
}

\begin{abstract}
Beckwith-Wiedemann syndrome (BWS) is a human genomic imprinting disorder that presents with a wide spectrum of clinical features, including overgrowth, abdominal wall defects, macroglossia, neonatal hypoglycemia, and predisposition to embryonal tumors. Its diagnosis is based on molecular tests or clinical signs. However, in children with features of BWS who do not fulfill the clinical diagnostic criteria, the molecular tests may play an important role in the diagnosis. There is an increased risk of embryonal tumors in patients with BWS, but few case reports have been reported on benign breast tumors in female adolescents with this syndrome. To our knowledge, this is the first case report in the literature that describes the imaging findings of fibroepithelial breast tumors (phyllodes tumor and fibroadenomas) in a 13-year-old female with BWS, highlighting the need for lifelong tumor surveillance in this patient population.
\end{abstract}

Keywords: Beckwith-Wiedemann syndrome, tumorigenesis, fibroepithelial breast tumors

Cite this article as: Oktay A, Esmat HA, Aslan Ö. Fibroepithelial Breast Tumors in a Teenager with Beckwith-Wiedemann Syndrome: A Case Report and Review of Literature. Eur J Breast Health 2021; 17(3): 288-291

\section{Key Points}

- Benign breast tumors can accompany to Beckwith-Wiedemann syndrome (BWS).

- Fibroepithelial tumor of the breast is a spectrum of lesions ranging from fibroadenoma to malignant phyllodes tumor.

- Lifelong tumor surveillance in patients with fibroepithelial tumor of breast in BWS is recommended.

\section{Introduction}

Beckwith-Wiedemann syndrome (BWS) is a human genomic imprinting disorder that presents with a wide spectrum of clinical features, including overgrowth, abdominal wall defects, macroglossia, neonatal hypoglycemia, and predisposition to embryonal tumors (1). It is a panethnic syndrome with a 1:1 sex ratio and approximate incidence of one in 10,000-13,700 births. Such incidence may be underestimated in mild phenotypes and most likely increases due to a positive correlation with assisted reproductive techniques. This syndrome is caused by diverse genetic and epigenetic disorders that usually affect the regulation of genes imprinted on chromosome $11 \mathrm{p} 15.5$ (2). The relevant imprinted chromosomal region in $11 \mathrm{p} 15.5$ and uniparental disomy (UPD) of chromosome $11 \mathrm{p} 15$ is a risk factor for BWS-associated tumorigenicity. Chromosome 11p15.5 consists of imprinting domains of IGF2, the expression of which is associated with the tumorigenesis of various breast cancers (3). To date, there have been only a few reports on benign breast tumors in patients with BWS. Here, the authors present a case of fibroepithelial breast tumors (phyllodes tumor and fibroadenomas) in a 13-year-old female with a known history of BWS to increase awareness about this rare and unusual presentation.

\section{Case Presentation}

A 13-year-old female with a known history of BWS presented to our hospital with a right breast mass in 2016. On physical examination, there were a right-sided hemihypertrophy (Figure 1), which she had since birth, and a nontender palpable large mass on the left breast, mobile over the underlying tissues, with no lymph nodes enlargement detected. Other findings were unremarkable. On an ultrasound, a giant solid mass measuring $7 \mathrm{~cm}$ in diameter was observed in her right breast. The mass was surgically excised, and the pathology result showed a juvenile 
fibroadenoma (Figure 2). In 2017, on a controlled ultrasound exam, no cystic or solid mass was detected in her breasts.

In 2018, on the breast ultrasound, it was found that she had multiple masses in the lower inner quadrant of the right breast (Figure 3). On magnetic resonance imaging images, multiple masses were observed in the lower quadrant of her right breast, with the largest one about $3 \mathrm{~cm}$ in diameter (Figure $4 \mathrm{a}$ and $\mathrm{b}$ ). The fine needle biopsy and cytology result of this lesion showed a benign entity. As this patient had a high risk of tumorigenesis and childhood cancers, following a multidisciplinary consultation, complex literature review, and evaluation of risks and

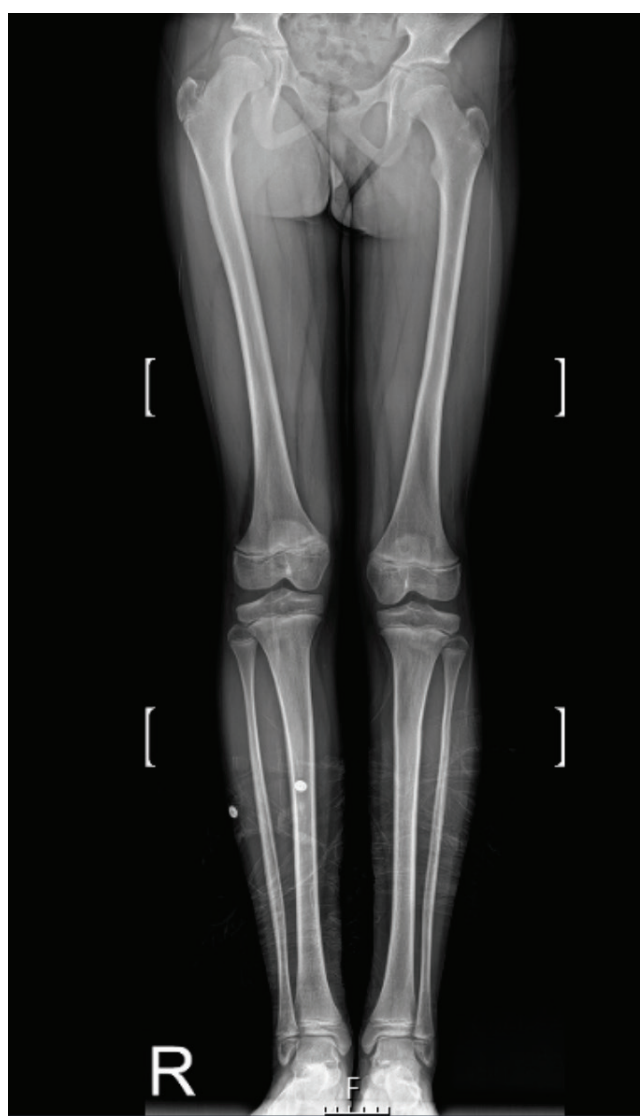

Figure 1. Plain $\mathrm{X}$-ray image of lower extremities showing a right femoral hemihypertrophy

R: Right benefits, a right breast lumpectomy was performed using a free nipple graft technique. The pathology examination of this lesion revealed a benign phyllodes tumor and fibroadenoma, but there were no signs of malignancy.

\section{Discussion and Conclusion}

BWS is the most common overgrowth syndrome. The condition was named after American pediatric pathologist John Bruce Beckwith in 1963, and German pediatrician Hans-Rudolf Wiedemann in 1964 , reported the syndrome independently (4). This syndrome is caused by diverse genetic and epigenetic disorders that usually affect the regulation of genes imprinted on chromosome 11p15.5 (2). It presents with a wide and varied clinical spectrum, including hemihyperplasia, macroglossia, prominent eyes with infraorbital creases, facial nevus flammeus, and midfacial hypoplasia, but the BWS facies often normalizes across childhood. Hyperinsulinemia, hypoglycemia, abdominal wall defects, visceromegaly, fetal adrenocortical cytomegaly, and renal and cardiac malformations are the important signs of this syndrome $(4,5)$.

The diagnosis is based on molecular tests or clinical signs. However, in children with features of BWS who do not fulfill clinical diagnostic criteria, the molecular tests may play an important role in diagnosis $(2,5)$. Our patient had BWS with a positive family history and characteristic right hemihypertrophy.

There is an increased risk of embryonal tumors in BWS, but few case reports have been published on benign breast tumors in female adolescents with this syndrome. These tumors were often asymmetric, and the applied medical strategy was their surgical removal (6).

To our knowledge, this is the first case report in the literature to describe asymmetric fibroepithelial breast tumors (phyllodes tumor and fibroadenomas) in a teenager with BWS. Fibroepithelial tumor of the breast is a heterogeneous group of lesions ranging from fibroadenoma at the benign end of the spectrum to malignant phyllodes tumor. There are overlapping histologic features among various subtypes, and transformation and progression to a more malignant phenotype may also occur (7). Fibroadenomas and phyllodes tumors share many common features. However, phyllodes tumors tend to show a more rapid growth and tend to recur if incompletely excised. Moreover, borderline and malignant phyllodes tumors may metastasize. In

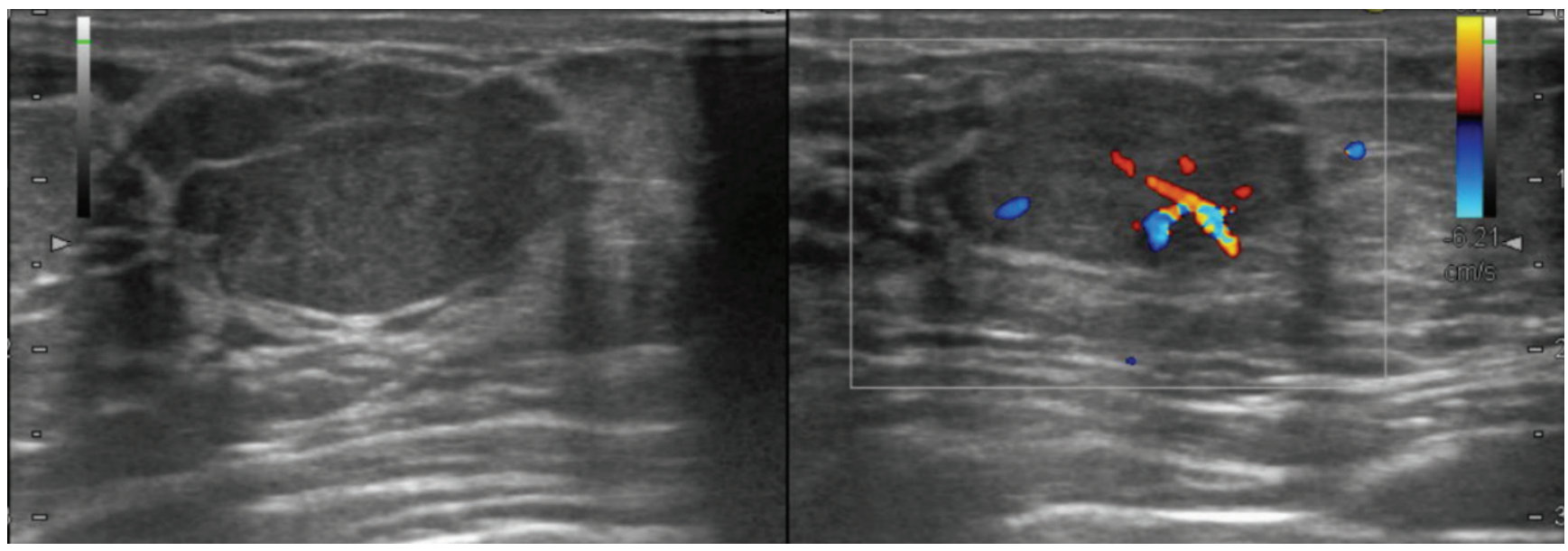

Figure 2. Ultrasound images of the right breast showing a well-defined, hypoechoic, lobulated mass with some vascularity, consisting of a fibroadenoma 


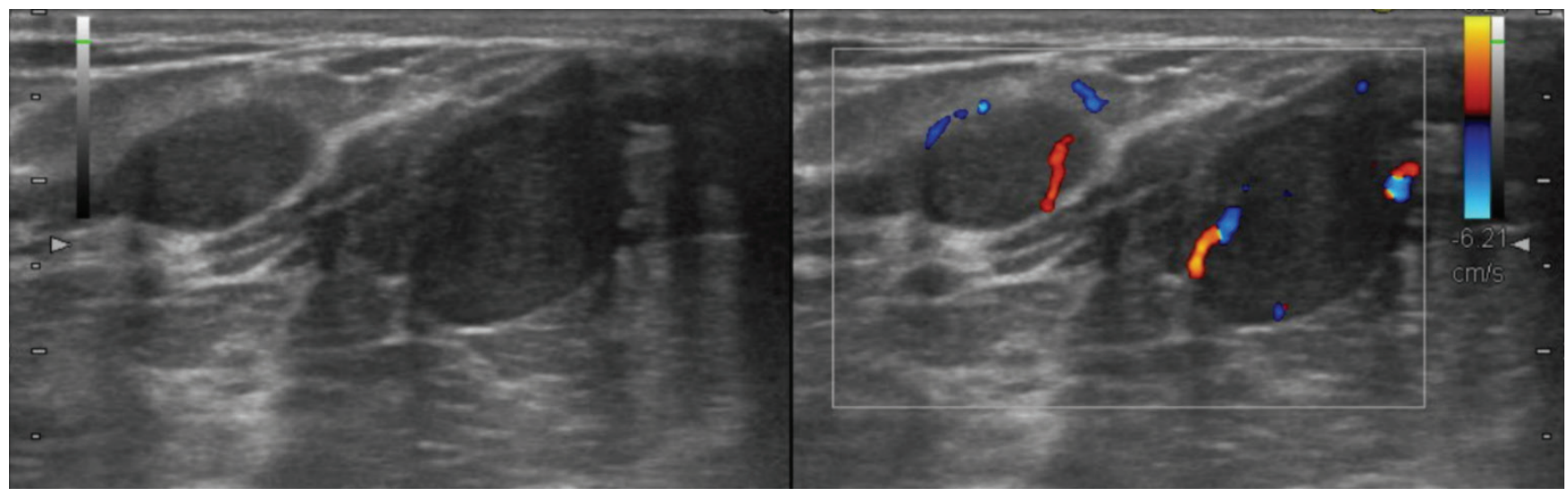

Figure 3. Ultrasound images of the right breast showing multiple well-defined, hypoechoic, lobulated masses with some vascularity, consisting of fibroepithelial breast tumors

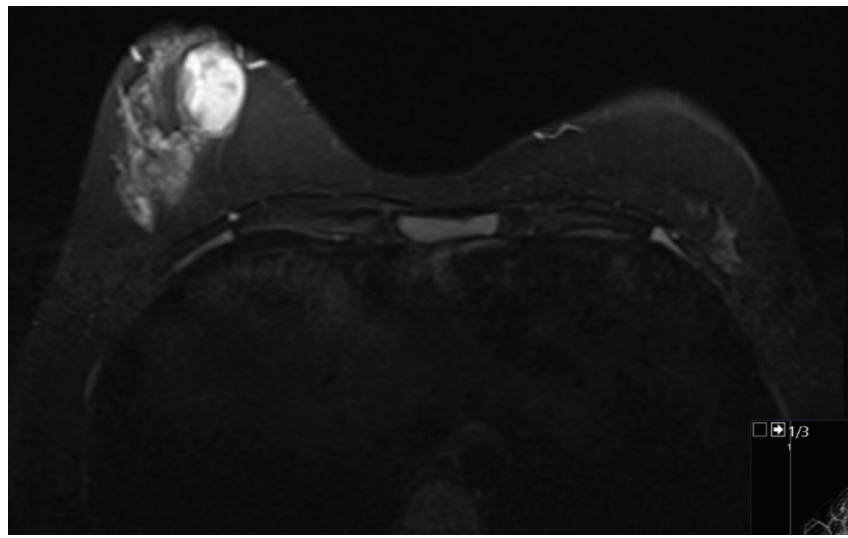

Figure 4a. Axial T2 weighted image demonstrates a high signal intensity mass with well-defined margins in the right breast

contrast, fibroadenomas usually need not be removed, and even when surgery is needed, enucleation is sufficient (8). In the patient described herein, a surgical reduction of giant breast lesion was performed using a free nipple graft technique, but the risk of recurrence may be the main lifelong concern Mishra et al. (9) in 2013. The relevant imprinted chromosomal region in $11 \mathrm{p} 15.5$ and UPD of chromosome $11 \mathrm{p} 15$ is a risk factor for BWS-associated tumorigenicity. Chromosome $11 \mathrm{p} 15.5$ consists of imprinting domains of IGF2, the expression of which is associated with the tumorigenesis of various breast cancers. However, it remains unclear whether BWS imposes an increased risk of breast lesions or a yet unknown molecular defect is responsible for the rare occurrence of this tumor in BWS (3-10).

This is the first case report in the literature to describe phyllodes tumor in a teenager with BWS. However, a wide range of both malignant and benign neoplasms has been reported in patients with BWS. This case report describes a case of fibroepithelial breast tumors (phyllodes tumor and fibroadenomas) in a 13-year-old female with BWS, highlighting the need for lifelong tumor surveillance in this patient population.

Informed Consent: Written informed consent was obtained from the patient's parents for publication of this case report.

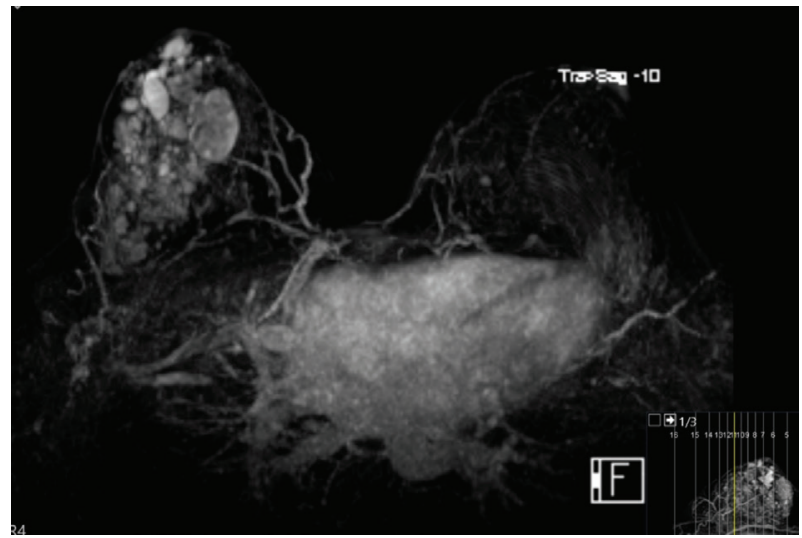

Figure 4b. 3D, maximum intensity projection (MIP), dynamic contrastenhanced subtracted MRI image showing multiple, well-defined masses in the right breast

MRI: Magnetic resonance imaging

\section{Authorship Contributions}

Conception: A.O.; Design: H.A.E.; Supervision: A.O.; Materials: Ö.A.; Data Collection and/or Processing: A.O., Ö.A.; Analysis and/or Interpretation: A.O.; Literature Search: Ö.A.; Writing: H.A.E.; Critical Review: A.O.

Conflict of Interest: The authors have no conflicts of interest to declare.

Financial Disclosure: The authors declare that this study received no financial support.

\section{References}

1. Wang KH, Kupa J, Duffy KA, Kalish JM. Diagnosis and Management of Beckwith-Wiedemann Syndrome. Front Pediatr 2020; 7: 562. (PMID: 32039119) [Crossref]

2. Cammarata-Scalisi F, Avendaño A, Stock F, Callea M, Sparago A, Riccio A. Beckwith-Wiedemann syndrome: clinical and etiopathogenic aspects of a model genomic imprinting entity. Síndrome de Beckwith-Wiedemann: aspectos clínicos y etiopatogénicos de una entidad ejemplo de impronta genómica. Arch Argent Pediatr 2018; 116: 368-373. (PMID: 30204990) [Crossref]

3. Takama Y, Kubota A, Nakayama M, Higashimoto K, Jozaki K, Soejima H. Fibroadenoma in Beckwith-Wiedemann syndrome with paternal uniparental disomy of chromosome 11p15.5. Pediatr Int 2014; 56: 931934. (PMID: 25521982) [Crossref] 


\section{Oktay et al. Fibroepithelial Breast Tumors in BWS}

4. Borjas Mendoza PA, Mendez MD. Beckwith Wiedemann Syndrome. (Updated 2020 Oct 1). In: StatPearls (Internet). Treasure Island (FL): StatPearls Publishing; 2020 Jan. (PMID: 32644419)

5. Weksberg R, Shuman C, Beckwith JB. Beckwith-Wiedemann syndrome. Eur J Hum Genet 2010; 18: 8-14. (PMID: 19550435) [Crossref]

6. Szymańska E, Moszczyńska E, Polnik D, Szymańska S, Jurkiewicz E, Pyzlak M, et al. Virginal breast hypertrophy in a patient with BeckwithWiedemann syndrome. Clin Case Rep 2018; 6: 484-489. (PMID: 29531723) [Crossref]

7. Yang X, Kandil D, Cosar EF, Khan A. Fibroepithelial tumors of the breast: pathologic and immunohistochemical features and molecular mechanisms. Arch Pathol Lab Med 2014; 138: 25-36. (PMID: 24377809) [Crossref]
8. Wiratkapun C, Piyapan P, Lertsithichai P, Larbcharoensub N. Fibroadenoma versus phyllodes tumor: distinguishing factors in patients diagnosed with fibroepithelial lesions after a core needle biopsy. Diagn Interv Radiol 2014; 20: 27-33. (PMID: 24356293) [Crossref]

9. Mishra SP, Tiwary SK, Mishra M, Khanna AK. Phyllodes tumor of breast: a review article. ISRN Surg. 2013; 2013: 361469. doi:10.1155/2013/361469 (PMID: 23577269) [Crossref]

10. Cappuccio G, De Crescenzo A, Ciancia G, Canta L, Moio M, Mataro I, et al. Giant breast tumors in a patient with Beckwith-Wiedemann syndrome. Am J Med Genet A 2014; 164A: 182-185. (PMID: 24214456) [Crossref] 